\title{
Monitoring distillation column systems using improved nonlinear partial least squares-based strategies
}

\author{
Muddu Madakyaru, Fouzi Harrou, Member, IEEE, Ying Sun
}

\begin{abstract}
Fault detection in industrial systems plays a core role in improving their safety, productivity and avoiding expensive maintenance. This paper proposed and veried datadriven anomaly detection schemes based on a nonlinear latent variable model and statistical monitoring algorithms. Integrating both the suitable characteristics of partial least squares (PLS) and adaptive neural network fuzzy inference systems (ANFIS) procedure, PLS-ANFIS model is employed to allow for flexible modeling of multivariable nonlinear processes. Furthermore, PLS-ANFIS modeling was connected with k-nearest neighbors (kNN)-based data mining schemes and employed for nonlinear process monitoring. Specifically, residuals generated from the PLS-ANFIS model are used as the input to the kNN-based mechanism to uncover anomalies in the data. Moreover, kNNbased exponentially smoothing with parametric and nonparametric thresholds is adopted to better anomaly detection. The effectiveness of the proposed approach is evaluated using real measurements from an actual bubble cap distillation column.
\end{abstract}

Index Terms-Anomaly detection, Data mining algorithm, Unsupervised monitoring, distillation column systems.

\section{INTRODUCTION}

$\mathbf{F}$ AULT detection in chemical or petrochemical processes provides relevant information for enhancing productivity, efciency, and safety in process industries. Generally, industrial processes are continuously exposed to failures and faults that are challenging to avoid and may lead to serious degradation of product quality, operation reliability, and profitability. In this context, the capacity to appropriately and timely detection of faults that may result in fatal accidents and substantial economic losses is of utmost importance. The role of fault detection, which is the focus of this paper, is to reveal any degradation and deviation from the process operation compared to its nominal condition [1], [2].

Detecting faults in industrial processes play a core role in developing efficient production systems that have acceptable performance and meet with the desired requirements and specifications. Hence, to quickly compare the performance of the inspected process to the requirements, various monitoring approaches have been designed in the literature [2], [3]. In general, there are two fault detection families: modelbased and data-derived methods. Model-based approaches use

M. Madakyaru is with Manipal Institute of Technology, Department of Chemical Engineering, Manipal Academy of Higher Education, Manipal, India

F. Harrou and Y. Sun are with King Abdullah University of Science and Technology (KAUST) Computer, Electrical and Mathematical Sciences and Engineering (CEMSE) Division, Thuwal, 23955-6900, Saudi Arabia e-mail: fouzi.harrou@kaust.edu.sa analytical models to uncover faults, which are not often easy to construct in particular for complex systems with a large number of variables. On another hand, data-based techniques carry information about the faults by extracting relevant features from data. Data-derived methods, nowadays are more commonly applied in engineering and petrochemical processes [4], [5]. For instance, fault detection in chemical process industries is challenging due to a large number of variables involved, the dynamic characteristics and noisy measurements in these processes. Indeed, a large number of variables leads to collinearity, which increases the uncertainty about the model parameter estimates. A very commonly used modeling framework to remedy such problems is the latent variable regression (LVR) models. LVR model can deal with collinearity among the variables, by constructing a model from a reduced number of variables (which are a linear combination of the original variables) called a latent variable or principal components. The approach results in well-conditioned models [1], [6]. The commonly used LVR model estimation techniques include principal component regression (PCR) [7], [8], partial least squares (PLS) [9]-[11], and regularized canonical correlation analysis [12].

Without an efficient detection procedure, chemical processes such as distillation columns would be damaged by unexpected faults and can result in nancial losses and serious damages. Frequently, linear latent variable regression models such as PCR and PLSR are used as tools for fault detection to monitor linear processes. However, the use of linear LVR methods is limited to capture the linear information in the data and monitor linear processes. Indeed, most of the practical data are non-linear in nature. To capture the nonlinearities in the data and monitor nonlinear processes, several data-driven methods are developed in the literature. For instance, the quadratic PLS proposed by Wold et al. [13], used to model the inner relation of the PLS model using a quadratic function. However, based only on a quadratic function may not provide enough flexibility to model complex systems. In a similar line, several nonlinear versions to model the inner relationship have been developed, such as neural network PLS [14], [15] and fuzzy PLS [16]. The method proposed in [15] uses the feed-forward neural network in the inner relation of the PLS to capture the nonlinearity in the data. The linear Takagi-Sugeno-Kang relations are used in inner relations of PLS to capture the nonlinearities [16]. The quadratic fuzzy function combined with PLS to achieve quadratic fuzzy PLS [17]. 
Since faults and failures are inevitable in engineering systems, they must be suitably detected and corrected. Indeed, fault detection in chemical process industries is major challenging due to a large number of variables involved and nonlinear behavior of the system. Also, these monitored variables are correlated among each other and associated with noise. In this paper, it is proposed to integrate the adaptive neural network fuzzy inference systems (ANFIS) procedure, the PLS modeling, and k-nearest neighbors ( $\mathrm{kNN}$ )-based data mining detector to improve efficiency nonlinear process monitoring.

The contributions of this study are as follows:

- The proposed approach introduces an improved model able to describe relevant features in multivariate inputoutput dynamic data by merging ANFIS and PLS, called PLS-ANFIS model. In the PLS-ANFIS model, ANFIS is employed to model the nonlinear inner relations linking the predictor and response LVs of the PLS model. PLSANFIS model is a very flexible input-output model able to capture nonlinear dependencies of large cross-correlated process variables. In other words, nonlinear PLS using the adaptive-network-based fuzzy inference system is used as an inner relation of the PLS model (i.e., mapping nonlinear relation between latent variable and output). This modeling framework method is based on constructing a set of fuzzy if-then rules with appropriate membership functions to approximate the relationships between the input-output pairs in the inner relation of the PLS model. A major advantage of adaptive neural network based fuzzy inference systems is that they adaptively optimize the parameters in the premise and consequent part of the fuzzy inference system. The PLS-ANFIS model is used to generate residuals for anomaly detection purpose.

- An additional novelty is to introduce an anomaly detection approach based on an unsupervised kNN-based procedure to distinguish normal from abnormal measurements. The major reason for using the $\mathrm{kNN}$ data mining approach to quantify the dissimilarity between normal and abnormal features is due to its capability to handle nonlinear features and without making assumptions on the underlying data distribution [18]. Here, a framework combining kNN and exponentially smoothing (ES) procedure is proposed for suitable fault detection. Indeed, the kNN-ES make use of all available information from past and actual measurements, which improve the detection abilities of the kNN-based scheme.

- Furthermore, the detection threshold of kNN-ES is computed non-parametrically via kernel density estimation, which extends the flexibility of the proposed approach. Residuals from the PLS-ANFIS model are employed as the input for the kNN-ES procedure for fault detection. The detection performances of the PLS-ANFIS-based kNN-ES approaches are evaluated using real distillation column measurements.

This paper is structured as follows. The PLS-ANFIS modeling approach is summarized in the next section. Then, the proposed PLS-ANFIS-based kNN procedure is described in
Section III. In Section IV presents the experimental results. Finally, conclusions are provided in Section V.

\section{PLS-ANFIS MOdELING Framework}

Consider an input with $n$ samples and $m$ variables $\mathbf{X} \in \mathbb{R}^{n \times m}$ and output with $n$ samples and $p$ variables $\mathbf{Y} \in \mathbb{R}^{n \times p}$. PLS extracts the principal components iteratively by maximizing the covariance of the extracted principal components. PLS model development has two components, one is to develop inner models and the other one is to develop outer models [3], [19]. Outer models have a relationship with the inner model as:

$$
\left\{\begin{array}{l}
\mathbf{X}=\sum_{i=1}^{l} \mathbf{t} \mathbf{t p}_{i}^{T}=\mathbf{T} \mathbf{P}^{T}+\mathbf{G} \\
\mathbf{Y}=\sum_{i=1}^{l} \mathbf{u} \mathbf{q}_{i}^{T}=\mathbf{U} \mathbf{Q}^{T}+\mathbf{F},
\end{array}\right.
$$

where, $\mathbf{T} \in \mathbb{R}^{n \times l}$ and $\mathbf{U} \in \mathbb{R}^{n \times q}$ represent a matrix of the transformed uncorrelated variables. The loading matrices of input and output space are $\mathbf{P} \in \mathbb{R}^{m \times l}$ and $\mathbf{Q} \in \mathbb{R}^{p \times q}$, respectively. The model residuals are the $\mathbf{G}$ and $\mathbf{F}$. Figure 1 illustrates a representative example of the core idea of PLS in wich $m=3$ ,$p=3$, and $l=1$

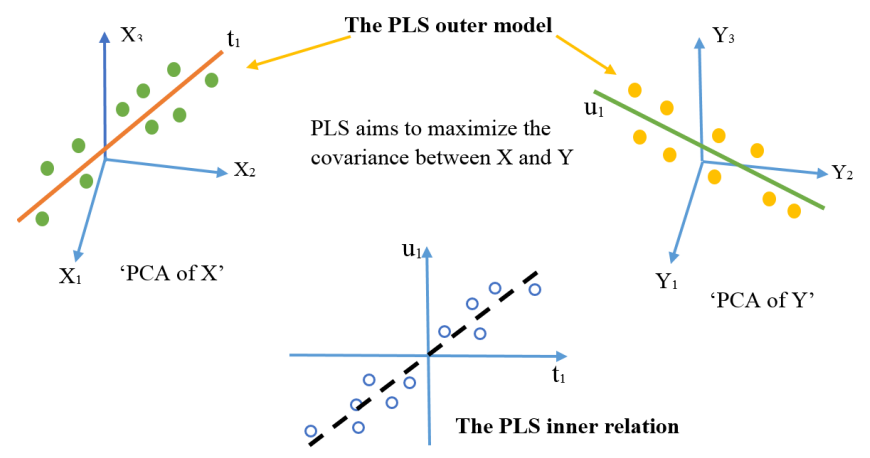

Fig. 1: Illustrative presentation of the core idea of PLS.

The number of principal components $(l)$ is determined by a well-known method called cross-validation. The retained latent variables of the input and output space are related by the nonlinear functions as:

$$
\mathbf{U}=f(\mathbf{T}, \Theta)+\mathbf{E},
$$

where $\Theta$ and $\mathbf{E}$ are the model parameters set and residue vector respectively (Figure 2).

To capture the nonlinear features in the data set in an environment with uncertainty, the fuzzy if-then rules are employed. If-then rules consist of a condition (also called a premise) and a result (called consequence). Several types of fuzzy if-then rules are available in the literature. Generally, fuzzy inference systems consist of fuzzy if-then rules, a decision-making unit, fuzzification step, and a de-fuzzification step. Fuzzy inference systems have been extensively used in system modeling and control applications. However, incorporating the human or expert knowledge into the rule base of the fuzzy inference system and tuning the membership functions have always been a challenge. These limitations are mitigated using adaptive neural network based fuzzy inference systems (ANFIS), in which the number and shapes of the membership function are 


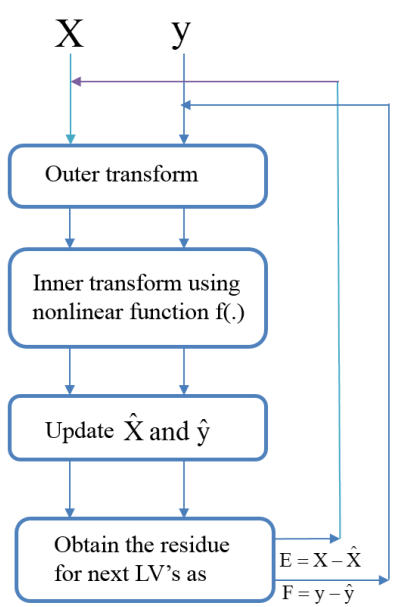

Fig. 2: General schematic representation of nonlinear PLS.

adaptively optimized to best fit the data. In this work, ANFIS is utilized to develop nonlinear PLS models.

ANFIS uses the neural network approach to the synthesis of the fuzzy inference system architecture. The idea behind using a neural network in fuzzy modeling is to first, mitigate the problem of transforming the human knowledge or expression into rule base of fuzzy inference system and second, to deal with effective methods for tuning the membership functions (MFs) of fuzzy inference system. The parameter set of an adaptive network node is the union of the parameter sets of each adaptive node. The structure of each node function may vary from node to node and choice of each node function depends on the overall input-output relation. Depending on the input-output relation, the adaptive nature of the network allows deciding the functions and determining the parameters in each node. ANFIS structure has five distinct layers which make the network multi-layer network. In the present study, the TSKtype fuzzy system with single-input single-output is used to capture the nonlinear relationship between the input and output latent space. The parameters of the fuzzy inference system for given input-output from the latent space are evaluated using a combination of least-squares and back-propagation gradient descent algorithm [20]. The network weights are trained such that the residual in equation (3), $\mathbf{e}_{i}$ is minimized.

$$
J_{i}=\left\|\mathbf{e}_{i}\right\|^{2}=\left\|\mathbf{U}_{i}-\mathbf{f}_{i}\left(\mathbf{T}_{i}, \Theta\right)\right\|^{2}
$$

The premise and consequent parameters of ANFIS are obtained in two passes for each epoch. In the first pass, the consequent parameters are determined using the least square method for a fixed value of premise parameters, this pass is called the forward pass. In the second pass, which is called the backward pass, the premise parameters are updated adaptively for a given value of consequent parameters and these parameters are obtained by back-propagation gradient descent algorithm. Briefly, the first layer in the ANFIS structure performs fuzzy formation and the second layer performs fuzzy rules. The third layer performs the normalization of membership functions and the fourth layer is the conclusive part of fuzzy rules and finally, the last layer determines the

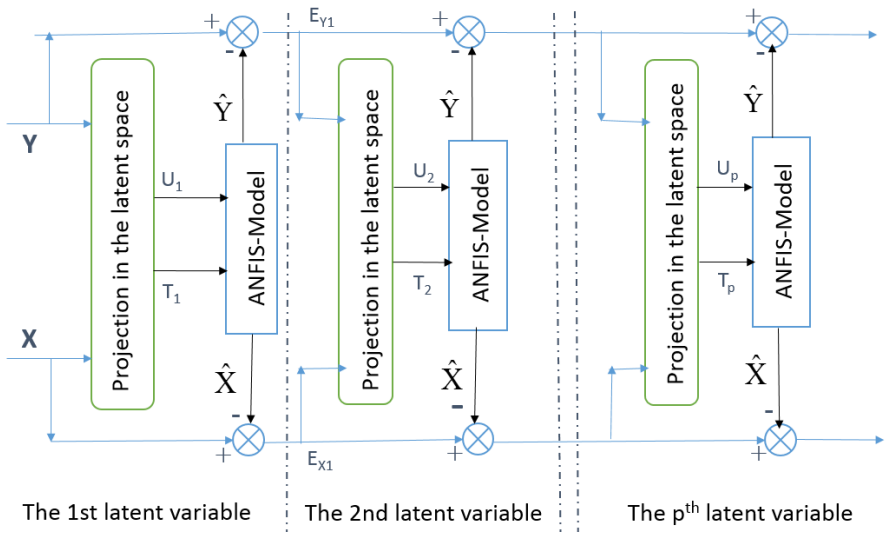

Fig. 3: Schematic illustration of PLS-ANFIS modeling.

network output.

1) PLS-ANFIS modeling algorithem: The proposed approach uses adaptive network-based FIS (ANFIS) as the inner regressors in PLS modeling. The nonlinear PLS based on the ANFIS [20]-[22] framework is schematically illustrated in Figure 3. The PLS outer transform is kept to generate latent variables in input and output space (i.e $\mathbf{T}_{i}$ and $\mathbf{U}_{i}$ ) from the data set. Then the latent variables from the input and output are used to train the inner network models. The latent variables $\left(\mathbf{T}_{i}\right)$ and $\left.\mathbf{U}\right)$ are used to estimate the adaptive-network-based fuzzy inference system parameters. The proposed PLS-ANFIS method differs from the direct network approach in that data are not directly used to train the networks, but are preprocessed by the PLS outer transform. Therefore this transform that decomposes the multivariate regression problem into a number of univariate regressors. Each regressor is implemented by adaptive network-based FIS. The optimal number of latent variables ' $p$ ' could be determined by cross-validation, which splits the latent space data into two data sets: one is for training, the other set is testing. The optimal number of latent variables selected such that the model gives the minimum prediction error for the testing set.

Figure 3 illustrates the schematic diagram of the PLSANFIS procedure. The ANFIS is used to capture the nonlinear relation between the score vectors $\left(\mathbf{T}_{i}\right)$ to model the inner relation by nonlinear function $\left(f_{L}().\right)$ of the PLS.

$$
\mathbf{u}=f(\mathbf{t}, \Theta)+\varepsilon
$$

The following steps describe the overall PLS-ANFIS procedure:

Step 1 The input $\mathbf{X}$ and output $\mathbf{Y}$ matrix converted into zero mean unit variance. let $\mathbf{E}=\mathbf{X}$ and let $\mathbf{F}=\mathbf{Y}$ and set $L=1$

Step 2 Project the input and output space in to the latent space and obtain $\mathbf{t}_{L}$ and $\mathbf{u}_{L}$

This step involves nonlinear partial least square (NIPALS) algorithm

Step 3 Apply ANFIS to capture the nonlinear relation $\mathbf{f}_{L}($.$) ,$ between output score $\mathbf{u}_{L}$ and latent variable $\mathbf{t}_{L}$ 
Step 4 Evaluate the loading vectors of $\mathbf{X}$ and output $\mathbf{Y}$

$$
\begin{aligned}
\mathbf{p}_{L}^{T} & =\frac{\mathbf{t}_{L} \mathbf{E}_{L-1}}{\mathbf{t}_{L}^{T} \mathbf{t}} \\
\mathbf{q}_{L}^{T} & =\frac{\hat{\mathbf{u}}_{L} \mathbf{F}_{L-1}}{\hat{\mathbf{u}}_{L}^{T} \hat{\mathbf{u}}}
\end{aligned}
$$

where $\hat{\mathbf{u}}=\mathbf{f}_{L}\left(\mathbf{t}_{L}\right)$

Step 5 Calculate the residue for the factor $L$

$$
\begin{gathered}
\mathbf{E}_{L}=\mathbf{E}_{L-1}-\mathbf{t}_{L} \mathbf{p}_{L} \\
\mathbf{F}_{L}=\mathbf{F}_{L-1}-\mathbf{u}_{L} \mathbf{q}_{L}^{T}
\end{gathered}
$$

Step 6 Let $L=L+1$, then return to the Step 2 until all the ' $p$ ' latent variables are calculated

\section{THE PROPOSED PLS-ANFIS-BASED KNN SCHEMES}

kNN classifier has been widely applied in the literature, however, needs the availability of labeled data for all the different types of faults [23]. However, for online monitoring, such data labeled data are not available. Here an unsupervised kNN-based monitoring approach is developed. In this section, PLS-ANFIS modeling is coupled together with the kNN data mining algorithm for enabling enhanced nonlinear process monitoring. As discussed above, nonlinear PLS using ANFIS is an effective approach for extracting relevant features from nonlinear multivariate input-output data. Here, PLS-ANFIS model is used with kNN for nonlinear process monitoring.

In the proposed approach, PLS-ANFIS is employed to describe the nonlinear process measurements and capture relevant features. Then residuals generated from the PLSANFIS model are fed to $k \mathrm{NN}$ for anomaly detection. The $\mathrm{kNN}$ is an unsupervised data mining approach and requires only the fault-free measurements for training without labeling.kNN does not make assumptions in the distribution of the data, which make it very flexible to compute the distance between the desired behavior and the actual behavior of the process under supervision. When $k \mathrm{NN}$ distance between a new unlabeled residual observation and the $k$-nearest neighbors of anomaly-free residuals is close to zero then the $k \mathrm{NN}$ detector signals these measurements as normal. Frequently, Euclidean distance was utilized to measure the closeness in $k \mathrm{NN}$-based methods [18], [24]. Large $\mathrm{kNN}$ distances are a signal of potential faults and accordingly used for process monitoring. To set up a decision threshold for monitoring, two kNN-based procedures are presented next.

In the proposed PLS-ANFIS-based kNN approach, the PLS-ANFIS model is implemented to describe nonlinearity in multivariate input-output processes. Based on fault-free measurements, the reference PLS-ANFIS model is designed and then adopted for fault detection. Indeed, after getting the reference PLS-ANFIS model, it is applied to new testing data to get residuals for fault detection. For new testing output measurements, $\mathbf{Y}=\left[y_{1}, \ldots, y_{t}, \ldots, y_{n}\right]$, the residual of the output variable, $\mathbf{E}=\left[e_{1}, \ldots, e_{t}, \ldots, e_{n}\right]$, are got via PLSANFIS model as,

$$
\mathbf{E}=\mathbf{Y}-\hat{\mathbf{Y}},
$$

where $\hat{\mathbf{Y}}=\left[\hat{y}_{1}, \ldots, \hat{y}_{t}, \ldots, \hat{y}_{n}\right]$ are the PLS-ANFIS predicted values. The $\mathrm{kNN}$-based detector is applied to residuals used to indicate an unusual shift from the reference PLS-ANFIS model. Specifically, the kNN approach is applied to residuals and construct the decision statistic for anomaly detection as described next. In the training stage, the $k \mathrm{NN}$ decision threshold, $H$, is computed based on fault-free measurements. In the testing phase, the distance between the new residuals and training residuals, $D_{\text {new }}$ is computed and compared to the computed threshold for fault detection. A block diagram of the proposed PLS-ANFIS-based kNN monitoring mechanism is given in Figure 4.

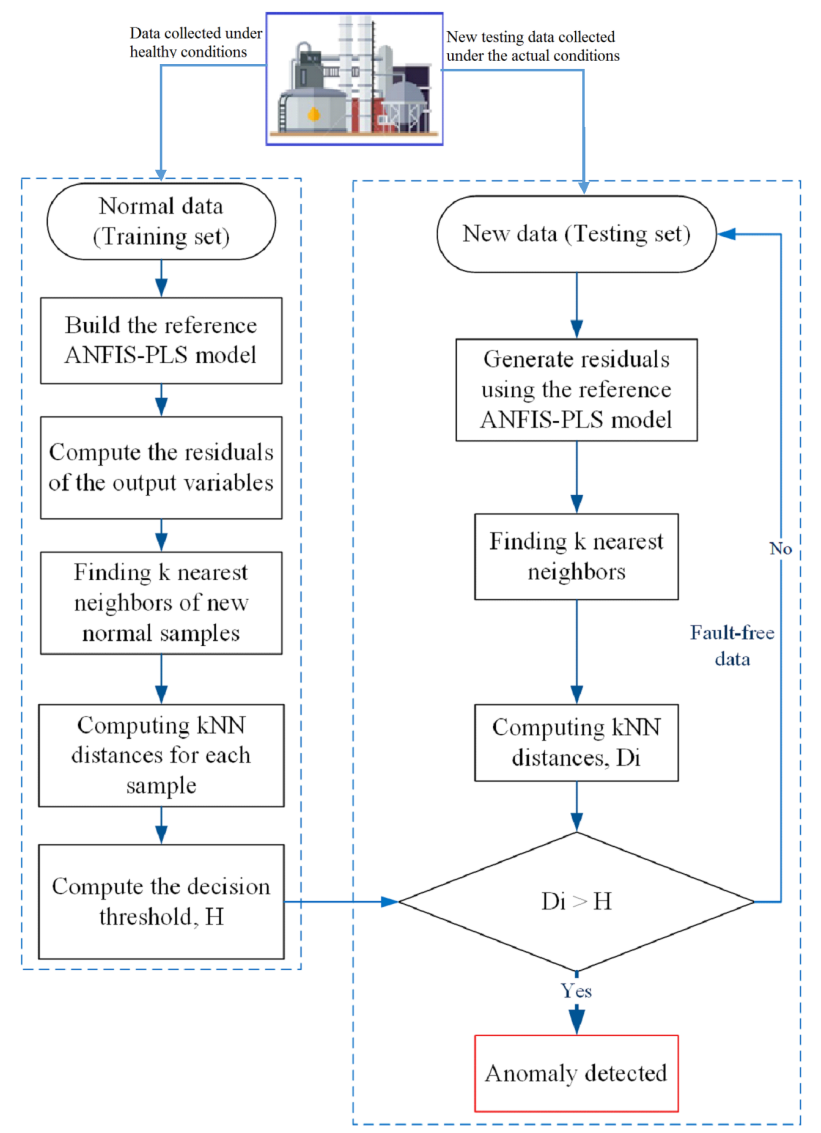

Fig. 4: Block diagram of PLS-ANFIS-based kNN monitoring mechanism.

\section{(1) $k N N-S h e w h a r t$ approach:}

Two monitoring charts are proposed in this studied to compute the decision threshold based on which faults can be detected. In the first approach, kNN-Shewhart, the kNN distances are evaluated using Shewhart chart to detect faults. It is performed by calculating the decision threshold using the three sigma rule.

$$
H=\mu_{D}+3 \sigma_{D},
$$

where $\mu_{D}$ and $\sigma_{D}$ are the mean and standard deviation of $k \mathrm{NN}$ distances using free-flow training measurements, and normality is assumed implicitly in this three-sigma methodology. 


\section{(2) $k N N-E S$ approach:}

The second approach is designed by applying the exponential smoothing (ES) chart to kNN distances. Indeed, kNN distances between the actual PLS-ANFIS residual observation and the normal training residual samples reflect inconsistencies between the healthy and faulty operating conditions. Then, the ES chart is used to evaluate kNN distances for fault detection. The $k$ NN-ES charting statistic is defined as follows:

$$
z_{d_{t}}=v d_{t}+(1-v) z_{d_{t-1}}
$$

where $z_{d_{0}}=\mu_{0}^{D}$ is the anomaly-free mean of the vector of $k \mathrm{NN}$ distances. $v(0<v 1)$ is the weighting factor. The value of $v$ is usually selected in the interval $0.1 \leq v \leq 0.3$ to detect small changes in practice [11], [25]. The $k$ NN-ES approach flags faults when the $k N N-E S$ statistic given in (11), $z_{t}^{k N N}$, is exceed the control limits, $h$, defined as

$$
h=\mu_{0}^{k N N}+\kappa \sigma_{0}^{k N N} \sqrt{\left(\frac{v}{(2-v)}\left[1-(1-v)^{2 t}\right]\right.},
$$

where $\kappa$ is a multiplier of standard deviation of $k \mathrm{NN}$ distances, $\sigma^{k N N}=\sigma_{0}^{k N N} \sqrt{\left(\frac{v}{(2-v)}\left[1-(1-v)^{2 t}\right]\right.}$.

The conventional parametric Shewhart and ES control procedures are suitable only when the normality assumption is valid. Indeed, the $k \mathrm{NN}$ algorithm is an efficient technique to identify the similarity between normal and abnormal features. However, associating it with a decision threshold based on the Gaussian distribution could degrade its performance. To bypass this limitation, the kernel density estimation [26] was used to estimate the distribution of $k \mathrm{NN}$ distances and compute the decision threshold nonparametrically for PLSANFIS-based fault detection. The main steps to implement kNN-based Shewhart and ES mechanisms with nonparametric thresholds are outlined next.

Step 1: For every input-output data, the residuals $E$ are obtained by the reference PLS-ANFIS model.

Step 2: Compute kNN distances of actual residuals to its nearest neighbor in the training set $D_{i}$, based on which sample distributions of distances can be obtained

Step 3: From the distribution of $D_{i}$, a nonparametric threshold of $k \mathrm{NN}-\mathrm{Shewhart}$ scheme is computed as the $(1-\alpha)$-th quantile of the estimated distribution of $k \mathrm{NN}$ distances obtained by KDE. Similarly, a nonparametric threshold of $k \mathrm{NN}-\mathrm{ES}$ approach is defined as $(1-\alpha)$-th quantile of the estimated distribution of the $k$ NN-ES statistic, $z$, obtained by KDE.

Step 4: Flag out traffic congestion when the kNN-based statistics ( $k \mathrm{NN}-$ Shewhart or $k \mathrm{NN}-\mathrm{ES})$ exceed the control limit.

\section{EXPERIMENTAL RESULTS AND DISCUSSION}

Here, the suitability of the PLS-ANFIS model to capture nonlinear and relevant features in multivariate input-output processes and the detection capacity of the kNN-based detectors are investigated. Real data from the distillation column is used to illustrate the designed detection schemes.

\section{A. Monitoring of experimental distillation column data}

The results of the experimental verification of the proposed fault detection strategy on a laboratory scale bubble cap distillation column are presented. The commonly used unit operation in the process industry for the purpose of separation of a component from the mixture of components is the bubble cap fractional distillation column. The used experimental setup of bubble cap distillation column is located at the Department of Chemical Engineering, Manipal Institute of Technology, Manipal Academy of Higher Education, Manipal, India. A pictorial view is shown in Figure 5.

The bubble cap fractional distillation unit is made up stainless steel 304 with a diameter of $100 \mathrm{~mm}$ rising up to 1.5 $\mathrm{m}$. The total number of trays in the column are 10 and two bubble caps fixed on each tray. The tray spacing is kept as 120 $\mathrm{mm}$, and each section of the individual tray are interconnected using mild steel flanges of $8 \mathrm{~mm}$ thick and with a rubber gasket. The inner details of bubble caps are with weirs of 25 $\mathrm{mm}$ height and a downcomer of $12 \mathrm{~mm}$ diameter are provided. The reboiler consists of a jacketed kettle of inside vessel dia is $100 \mathrm{~mm}$ diameter and $250 \mathrm{~mm}$ height and made up of stainless steel 304. To condense the vapor, the Shell \& Tube overhead condenser is used with $100 \mathrm{~mm}$ diameter of a shell and 450 $\mathrm{mm}$ long and is made of stainless steel 304 material. The tube bundle of shell \& tube heat exchanger consists of 7 tubes with $10 \mathrm{~mm}$ diameter and $450 \mathrm{~mm}$ long made up of stainless steel. The whole column is insulated to avoid heat loss. The re-boiler is fitted with a $2.0 \mathrm{~kW}$ heater to regulate the heat duty. The power to the heater is regulated using a thyristor controller that accept $4-20 \mathrm{~mA}$ input. The reflux flow and feed flow rate can be regulated using rate controlled metering pumps. Six resistance temperature detectors (RTD) sensors fixed at different locations of the column to monitor the distillation column temperatures, feed, and coolant fluid temperatures. The bubble cap distillation test set-up is interfaced with a control computer using a data acquisition system. The manipulated variables such as reflux flow and re-boiler heat duty are regulated to keep the top and bottom product composition at the desired level. In this experiment, a binary mixture of the methanol-water system has been used for distillation.

1) Data generation: To develop a model and test the proposed fault detection algorithm, the distillation column dataset is used. The distillation column dataset is generated by perturbing the column around its nominal operating condition. The perturbation data set is then split into training and testing data set. The training set is used for model development which is free from any faults and testing data set which contains faults are used to find the efficacy of the proposed fault detection mechanism. First, the distillation column is brought to normal operating condition and then the feed flow is perturbed with the magnitude of \pm 50 while keeping the reflux flow constantly at the nominal condition. Once the column comes to normal condition again distillation column is perturbed with reflux flow with the magnitude of \pm 40 and keeping the flow rate constant. The corresponding changes in the distillate composition i.e., methanol (xD) is shown in the Figure 6 and this is considered as the output variable. The 


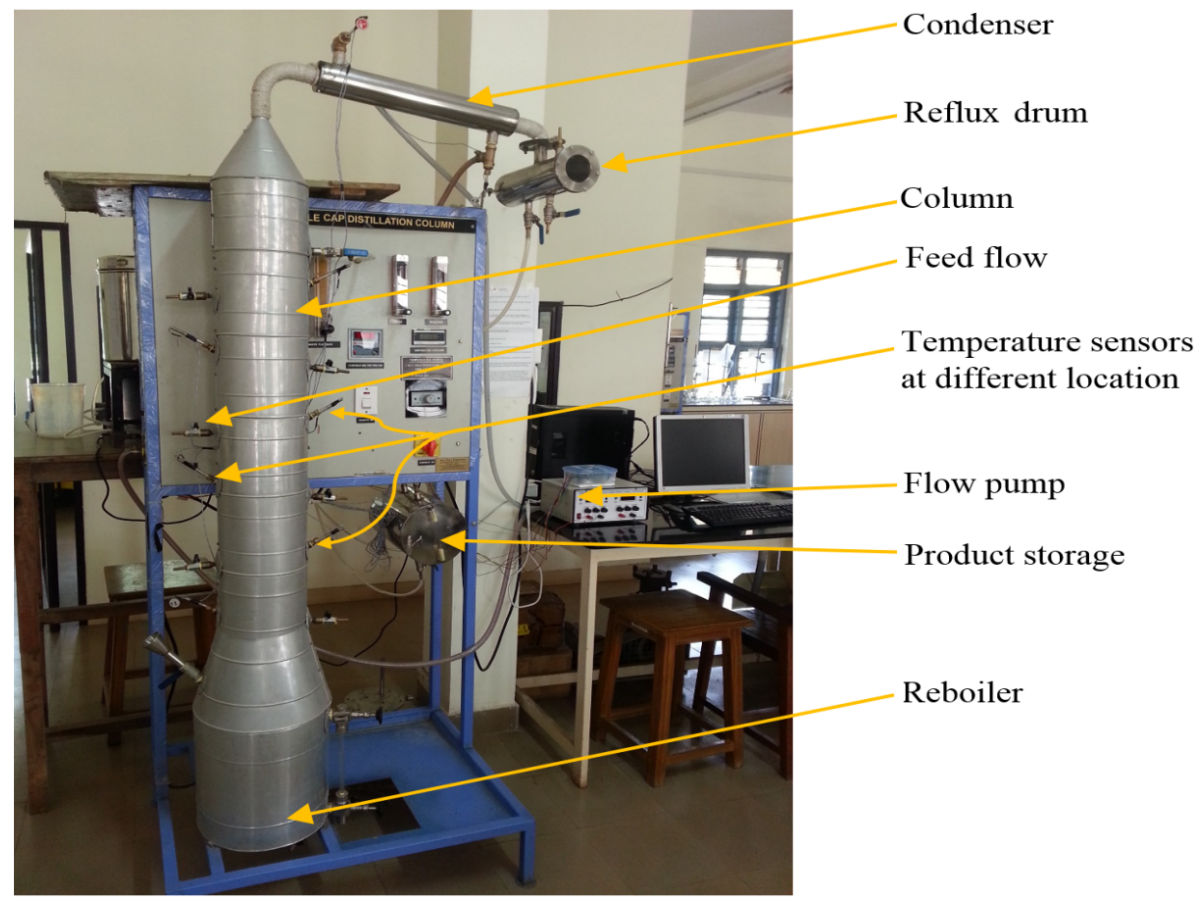

Fig. 5: Experimental setup: Bubble Cap Distillation Column.

sampling time chosen in this study is 4 sec. In the model development stage, along with flow rate and feed flow, six temperatures are included in the input matrix. The composition of the light distillate (methanol) i.e., $\mathrm{xD}$ is considered as the output variable.

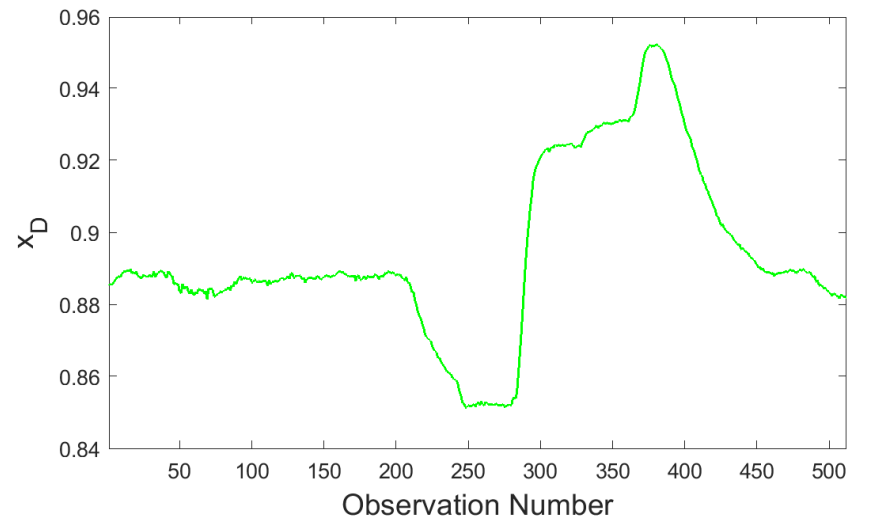

Fig. 6: Variation of the quality variable, $\mathrm{xD}$ (concentration of methanol in the distillate) for changes in the reflux flow.

PLS-ANFIS model is used to represent the nonlinear relation between the inner model of the PLS part, i.e., between the latent variable and the output. PLS-ANFIS generates an initial Sugeno-type fuzzy inference system (FIS) using a grid partition on the data between the first latent variable and the output. The initial FIS provides the initial condition to train the FIS. To, train the PLS-ANFIS, the above FIS is provided with Gaussian membership function with five fuzzy rules for each latent variable. Each rule generates an output function with linear mapping to the output. The trained PLS-ANFIS uses the hybrid algorithm to identify the membership function parameters, i.e., a combination of backpropagation and least squares methods are used for the training the FIS membership function. In this case, the total generation number and mean square error were set to 500 and 0.001 respectively. Then the antecedent and consequent parameters of the ANFIS network were tuned.

The designed PLS-ANFIS model that reflects the healthy behavior of the distillation column will be used for fault detection purposes. Distillation column processes are frequently exhibited to faults and failures that can degrade their desired specifications and performances. Four additive faults are considered in this study to test the performance of the proposed schemes: sensor bias, drift fault, intermittent fault, and sensor freezing fault. The sensor abrupt anomaly can be simulated as an additive step change. Intermittent faults appear and disappear repetitively for a small range of time. Sensor drift, which can reflect the sensor aging and slow degradation, can be simulated as a time-varying ramp signal. The sensor freezing can happen when the sensor output remains fixed at a particular value. To assess quantitatively the detection efficiency of the proposed procedures, the following metrics will be used: true positive rate (TPR), false positive rate (FPR), accuracy, and area under the curve (AUC). A confusion matrix and equations of the main related metrics that are frequently utilized to assess the quality of binary decision methods are summarized in Figure 7.

\section{(A) Scenario with sensor abrupt anomaly}

To test the detection capacity of the proposed techniques, 524 samples gathered from the distillation column are used for testing. In this scenario, it is considered that the temperature sensor $T_{3}$ is abruptly failed from observation 200 to 300 . Three cases were conducted to verify the feasibility of the detection mechanisms in the presence of abrupt anomalies. In the first 


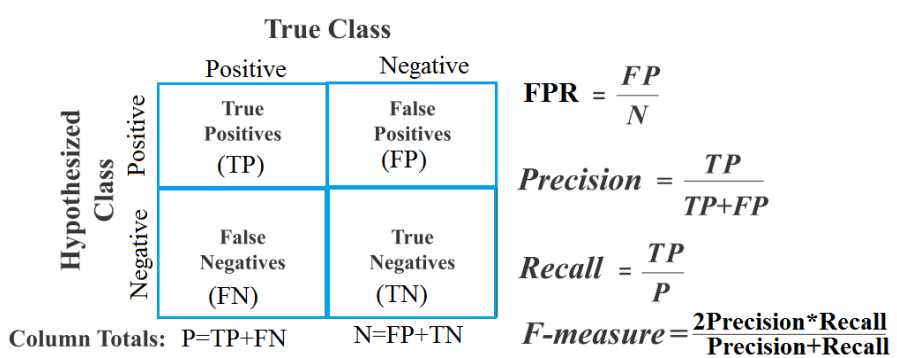

Fig. 7: Confusion matrix and associated commonly used performance metrics.

case, to simulate abrupt fault, a moderate bias of $15 \%$ of the total variation in the raw measurements is incorporated in the raw measurements. Detection results of the four monitoring schemes are summarized in Table I. As shown in Table I, the four methods shown good performances by detecting this anomaly with an AUC greater than 0.92, since this anomaly is relatively large. In addition, the results show that the use of nonparametric thresholds improves the detection accuracy of the kNN-based approach by achieving AUC around 0.96.

TABLE I: Summary of detection metrics for every monitoring scheme when sensor abrupt anomaly has happened (15\%).

\begin{tabular}{l|l|l|r|r|l}
\hline & TPR & FPR & Accuracy & F1Score & AUC \\
\hline KNN-ES $^{\text {np }}$ & 0.99 & 0.063 & 0.947 & 0.880 & 0.963 \\
\hline KNN-ES $^{p}$ & 0.99 & 0.054 & 0.955 & 0.896 & 0.968 \\
\hline KNN-Shewhart $^{\text {tp }}$ & 0.93 & 0.010 & 0.978 & 0.944 & 0.960 \\
\hline KNN-Shewhart $^{p}$ & 0.82 & 0.002 & 0.963 & 0.896 & 0.909 \\
\hline
\end{tabular}

In the second case, a bias of $10 \%$ is injected in the test data. According to Table II, kNN-based ES and Shewhart with nonparametric thresholds provide superior detection compared to their parametric counterparts.

TABLE II: Summary of detection metrics for every monitoring scheme when sensor abrupt anomaly has happened (10\%).

\begin{tabular}{l|l|l|r|r|r}
\hline & TPR & FPR & Accuracy & F1 Score & AUC \\
\hline KNN-ES $^{n p}$ & 0.99 & 0.085 & 0.930 & 0.846 & 0.952 \\
\hline KNN-ES $^{p}$ & 0.91 & 0.019 & 0.967 & 0.915 & 0.945 \\
\hline KNN-Shewhart $^{\text {pp }}$ & 0.99 & 0.131 & 0.892 & 0.783 & 0.929 \\
\hline KNN-Shewhart $^{p}$ & 0.86 & 0.005 & 0.969 & 0.915 & 0.928 \\
\hline
\end{tabular}

In the third case, a small bias of 5\% is injected in the temperature sensor $T_{3}$ test data. Detection performances of the designed detectors are given in Table III. Table III indicates that the kNN-Shewhart using a threshold based on the Gaussian distribution results in poor detection performance (i.e., $\mathrm{AUC}=0.583$ and $\mathrm{TPR}=0.17$ ). The use of $\mathrm{kNN}$-Shewhart with a nonparametric threshold improve the slightly the detection efficiency compared to its parametric counterpart (i.e., $\mathrm{AUC}=$ 0.716 and $\mathrm{TPR}=0.47$ ). Indeed, the $\mathrm{kNN}$-Shewhart provides acceptable results in the presence of moderate and large changes, but it cannot be used to detect small changes. The decision rule of kNN-Shewhart is based only on the actual observation and ignore the information in the past data, which make it inefficient for detecting small changes.
TABLE III: Summary of detection metrics for every monitoring scheme when sensor abrupt anomaly has happened (5\%).

\begin{tabular}{l|l|l|r|r|l}
\hline & TPR & FPR & Accuracy & F1Score & AUC \\
\hline KNN-ES $^{\text {np }}$ & 0.87 & 0.085 & 0.906 & 0.784 & 0.892 \\
\hline KNN-ES $^{p}$ & 0.86 & 0.085 & 0.904 & 0.778 & 0.887 \\
\hline KNN-Shewhart $^{\text {tp }}$ & 0.47 & 0.039 & 0.865 & 0.577 & 0.716 \\
\hline KNN-Shewhart $^{p}$ & 0.17 & 0.005 & 0.834 & 0.286 & 0.583 \\
\hline
\end{tabular}

As expected, the kNN-ES method (parametric and nonparametric) leads to improved anomaly detection compared the kNN-Shewhart (Table III). Using the parametric and nonparametric kNN-ES methods the obtained AUC are 0.892 and 0.887 , respectively. According to Table III, the kNN-ES with a nonparametric threshold exhibits superior detection over other approaches. Monitoring based on the kNN-ES scheme results in improved detection quality by increasing the detection rate (i.e., $\mathrm{AUC}=0.892$ and $\mathrm{TPR}=0.87$ ).

Figure 8 summarizes the AUC values of the four approaches for different congestion magnitudes. Figure 8 indicates that nonparametric kNN-ES provides better detection efficiency compared to the other approaches in particular for faults with a small magnitude. This is mainly due to the use of the parametric threshold and considering all information from past and actual data.

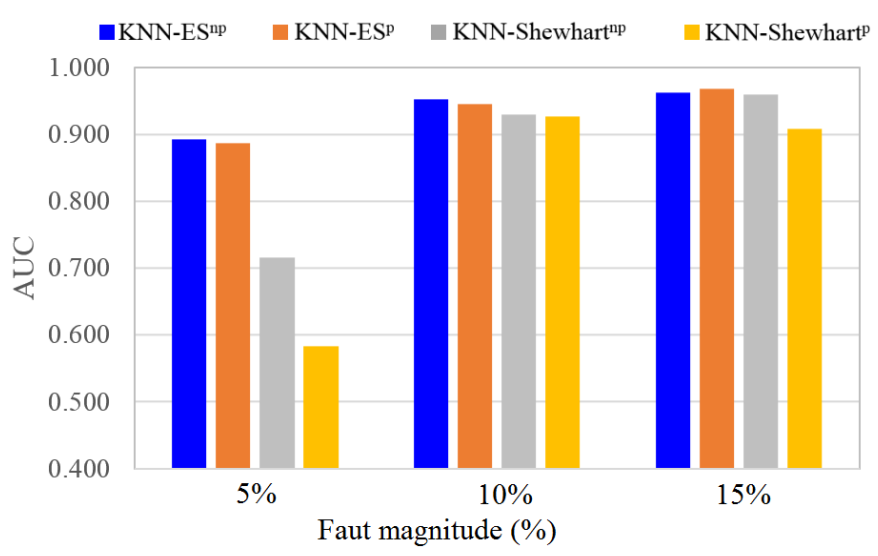

Fig. 8: AUC of kNN-based procedures for different fault magnitudes.

\section{(B) Scenario with sensor intermittent anomaly}

The target of this case is to test the detection capability of the PLS-ANFIS-based kNN schemes when intermittent faults happened. This case focuses on testing the capability of the designed mechanisms in sensing intermittent faults. To do so, intermittent anomalies with amplitude $7 \%$ of the total variation of raw measurements of $\mathrm{T} 3$ are added to the testing measurements for samples 100 to 150 , and for samples 300 to 350 . Table IV illustrates the obtained detection performance for kNN-based ES and Shewhart mechanisms. Table IV highlights the superiority of the nonparametric kNN-based ES scheme with lower FPR and the highest TPR, accuracy, and precision. The nonparametric kNN-ES scheme achieved the highest performance with an AUC of 0.961 (Table IV). 
It should be also pointed out that the parametric kNN-ES and kNN-Shewhart (parametric and nonparametric) schemes achieved respectively an AUC of 0.956, 0.901, 0.896, they flag this intermittent anomaly but with some false alarms and missed detection (Table IV). As anticipated, the nonparametric kNN-ES scheme shows better performance compared to the other algorithms (Table IV).

TABLE IV: Summary of detection metrics for every monitoring scheme when sensor intermittent anomaly has happened.

\begin{tabular}{l|l|l|r|r|r}
\hline & TPR & FPR & Accuracy & F1Score & AUC \\
\hline KNN-ES $^{\text {np }}$ & 0.961 & 0.039 & 0.961 & 0.907 & 0.961 \\
\hline KNN-ESp & 0.961 & 0.049 & 0.953 & 0.891 & 0.956 \\
\hline KNN-Shewhart $^{\mathrm{pp}}$ & 0.980 & 0.178 & 0.853 & 0.727 & 0.901 \\
\hline KNN-Shewhart $^{\mathrm{p}}$ & 0.980 & 0.188 & 0.845 & 0.717 & 0.896 \\
\hline
\end{tabular}

The above results in Table IV are obtained when using kNN with Euclidian and $k=1$. To investigate the impact of the used distance and the number of neighbors on the detection performance of kNN-based algorithms, we implemented the four schemes using Euclidian distance, Manhattan distance, and Chebychev distance when the number of neighbors used to compute kNN distance changes from 1 to 4 . Figure 9 (a-c) displays the AUC values of the four approaches when using Euclidian distance, Manhattan distance, and Chebychev distance, respectively. The peak AUC reached by the proposed kNN-ES approach with a nonparametric threshold (AUC $=0.991)$ when using kNN with Manhattan distance and $k=3$. Also, results indicate that nonparametric kNN-ES provides better detection efciency compared to the other approaches. On the other hand, the highest AUC by kNN-ES with Euclidian distance is obtained for $k=3$ ( $\mathrm{AUC}=0.983$ ), and with Chebychev $\mathrm{AUC}=0.984$ for $k=2$. Moreover, it is demonstrated enhanced performance are obtained when considering more neighbors than one in the computation of $\mathrm{kNN}$ distance.

\section{(C) Scenario with sensor freeze anomaly}

To further illustrate the capacity of the designed detectors, in this scenario, we consider a sensor freeze fault. For this purpose, the value of the temperature sensor $\mathrm{T} 3$ is fixed (equal to the observation value at time 300) from observation 300 until the end of the test measurements. Table $\mathrm{V}$ displays the detection results of the four kNN-based mechanisms. As the occurred anomaly breaks the cross-correlation among process variables, the four schemes provide good detection with an AUC greater than 0.93. First, results in Table V show that the kNN-based ES and Shewhart with the nonparametric thresholds provide slightly improved detection results compared to the corresponding Gaussian distribution-based thresholds. Table $\mathrm{V}$ confirm also the outperformance of the nonparametric kNN-ES mechanism compared to the other charts. Anomaly detection using the kNN-ES mechanism improves the detection quality by reaching an AUC of 0.973 , and lower FPR. These results confirm the advantage of the kNN-ES mechanism over other methods. This highlights the benefit of exponentially smoothing $\mathrm{kNN}$, which permit including all available information from past and actual data in the decision process. Also, from this case study, it can be concluded that

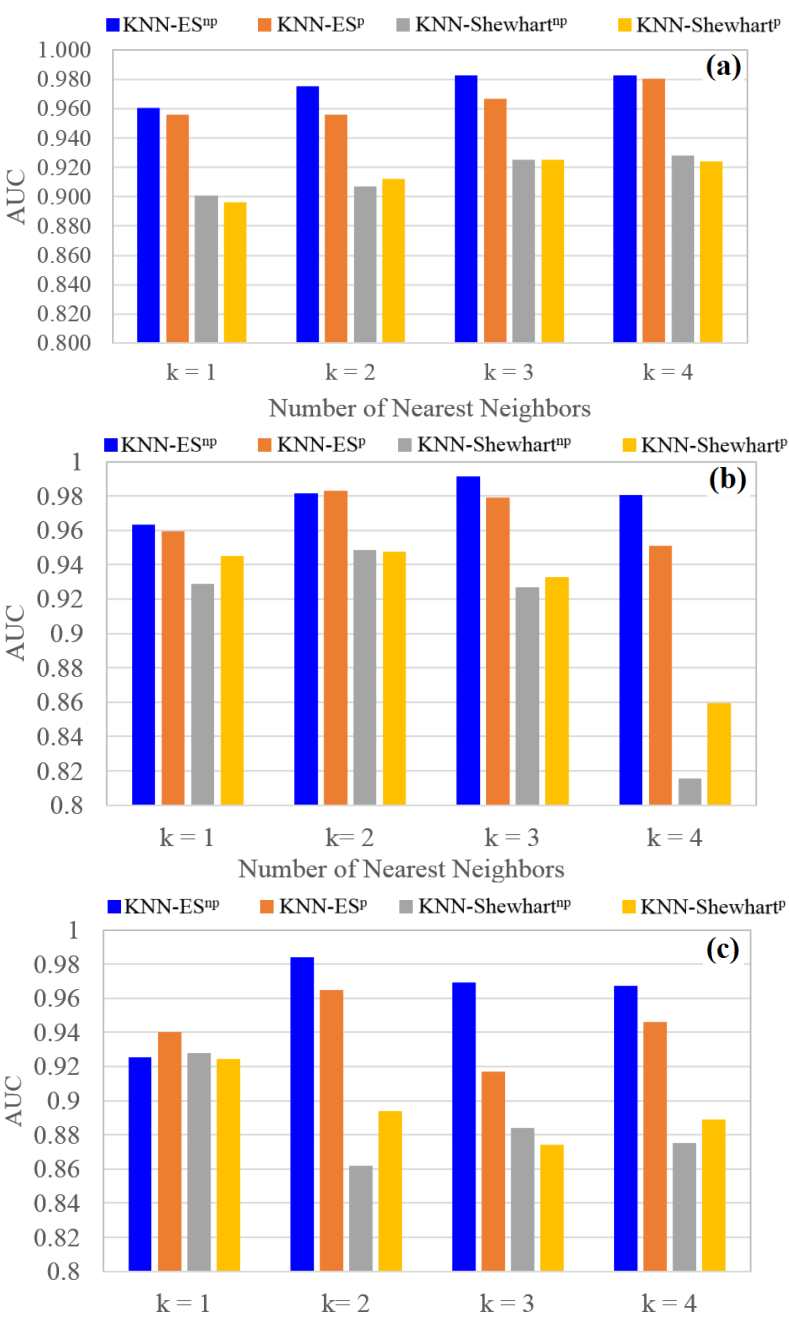

Fig. 9: AUC of kNN procedures as a function of the numbers of neighbors when using (a) Euclidian distance, (b) Manhattan distance, and (c) Chebychev distance.

kNN-based ES and Shewhart mechanisms with nonparametric thresholds computed via KDE provide improved detection performance compared to their parametric thresholds-based counterparts.

TABLE V: Summary of detection metrics for every monitoring scheme when sensor freeze anomaly has happened.

\begin{tabular}{l|r|l|r|r|r}
\hline & \multicolumn{1}{|l|}{ TPR } & FPR & Accuracy & F1Score & AUC \\
\hline KNN-ES $^{\text {np }}$ & 1 & 0.053 & 0.969 & 0.963 & 0.973 \\
\hline KNN-ESp & 1 & 0.073 & 0.957 & 0.950 & 0.963 \\
\hline KNN-Shewhart $^{\mathrm{np}}$ & 0.929 & 0.043 & 0.945 & 0.933 & 0.943 \\
\hline KNN-Shewhart $^{\mathrm{p}}$ & 0.900 & 0.037 & 0.937 & 0.922 & 0.932 \\
\hline
\end{tabular}

\section{(D) Scenario with Drift sensor fault:}

Here, the aim is to illustrate the potential of the designed schemes in sensing sensor drift faults. In this regard, early detection of drift faults before they propagate and seriously degrade the process is essential. For the experiment, a 5\% drift fault is injected in the temperature sensor, $T C_{3}$, at observation 300. Table VI displays the monitoring performance of the 
k-NN-based algorithms. According to Table VI, the kNNES method detected more accurately the progressive anomaly than the other approaches. The monitoring performance of the kNN-ES detector with the nonparametric threshold was better than the kNN-Shewhart schemes and the kNN-ES with a parametric threshold.

TABLE VI: Summary of detection metrics for every monitoring scheme when sensor intermittent anomaly has happened.

\begin{tabular}{l|r|r|r|r|l}
\hline & \multicolumn{1}{|c|}{ TPR } & FPR & Accuracy & F1Score & AUC \\
\hline KNN-ES $^{\text {np }}$ & 1 & 0.047 & 0.973 & 0.968 & 0.977 \\
\hline KNN-ES $^{p}$ & 1 & 0.07 & 0.959 & 0.953 & 0.965 \\
\hline KNN-Shewhart $^{\text {np }}$ & 0.938 & 0.017 & 0.965 & 0.957 & 0.961 \\
\hline KNN-Shewhart $^{\mathrm{p}}$ & 0.953 & 0.043 & 0.955 & 0.946 & 0.955 \\
\hline
\end{tabular}

In sum, this study indicates that the designed PLS-ANFISbased kNN mechanism exhibits a satisfactory capacity in identifying abrupt, intermittent, freeze and drift sensor faults in distillation columns. Furthermore, results proclaim the superior efficiency of the nonparametric kNN-based ES and Shewhart algorithms compared to the parametric ones. Also, results highlighted the superior performance of the kNN-ES in detecting small changes.

\section{CONCLUSION}

This paper presented an integrated statistical mechanism merging the benefits of PLS, ANFIS modeling and the kNNbased data mining scheme for nonlinear process monitoring. The proposed approach relies on the flexibility of the PLSANFIS model to capture relevant features in multivariate input-output processes and the good capacity of an unsupervised $\mathrm{kNN}$-based procedure to distinguish normal from abnormal measurements. The PLS-ANFIS is performed to generate residuals for fault detection. For the purpose of anomaly detection, the exponentially smoothed kNN (ES$\mathrm{kNN}$ ) is applied to residuals generated by the PLS-ANFIS model. A nonparametric threshold of the ES-kNN detector is computed kernel density estimation method. This provides more flexibility to the ES-kNN detector and the absence of assumption about the distribution underlying the data. The detection ability of the proposed approach is checked using real measurements from a pilot plant distillation column. The results demonstrate that the proposed schemes are effective in supervising nonlinear processes. As a future work, the proposed approach will be extended to consider the multiscale nature of processes based on multiresolution representation via wavelets.

\section{ACKNOWLEDGMENT}

The work presented in this publication was supported by the King Abdullah University of Science and Technology (KAUST) Office of Sponsored Research (OSR) under Award No: OSR-2019-CRG7-3800.

\section{REFERENCES}

[1] S. Yin, S. X. Ding, X. Xie, and H. Luo, "A review on basic data-driven approaches for industrial process monitoring," IEEE Transactions on Industrial Electronics, vol. 61, no. 11, pp. 6418-6428, 2014.
[2] F. Harrou, L. Fillatre, and I. Nikiforov, "Anomaly detection/detectability for a linear model with a bounded nuisance parameter," Annual Reviews in Control, vol. 38, no. 1, pp. 32-44, 2014.

[3] S. Qin, "Survey on data-driven industrial process monitoring and diagnosis," Annual Reviews in Control, 2012.

[4] F. Harrou, M. Madakyaru, Y. Sun, and S. Khadraoui, "Improved detection of incipient anomalies via multivariate memory monitoring charts: Application to an air flow heating system," Applied Thermal Engineering, vol. 109, pp. 65-74, 2016.

[5] F. Harrou, M. Madakyaru, and Y. Sun, "Improved nonlinear fault detection strategy based on the hellinger distance metric: Plug flow reactor monitoring," Energy and Buildings, vol. 143, pp. 149-161, 2017.

[6] T. Cheng, F. Harrou, Y. Sun, and T. Leiknes, "Monitoring influent measurements at water resource recovery facility using data-driven soft sensor approach," IEEE Sensors Journal, vol. 19, no. 1, pp. 342-352, 2018.

[7] I. Frank and J. Friedman, "A statistical view of some chemometric regression tools," Technometrics, vol. 35, no. 2, pp. 109-148, 1993.

[8] G. Wang and J. Jiao, "Quality-related fault detection and diagnosis based on total principal component regression model," IEEE Access, vol. 6, pp. 10341-10347, 2018.

[9] R. Muradore and P. Fiorini, "A PLS-based statistical approach for fault detection and isolation of robotic manipulators," IEEE Transactions on Industrial Electronics, vol. 59, no. 8, pp. 3167-3175, 2012.

[10] S. Yin, S. Ding, a. H. H. A. Haghani, and P. Zhang, "A comparison study of basic data-driven fault diagnosis and process monitoring methods on the benchmark Tennessee Eastman process," Journal of Process Control, vol. 22, no. 9, pp. 1567-1581, 2012.

[11] F. Harrou, Y. Sun, M. Madakyaru, and B. Bouyedou, "An improved multivariate chart using partial least squares with continuous ranked probability score," IEEE Sensors Journal, vol. 18, no. 16, pp. 67156726, 2018.

[12] S. S. D. R. Hardoon and J. Shawetaylor, "Canonical correlation analysis: An overview with application to learning methods," Neural Comput., vol. 16, no. 12, pp. 2639-2664, 2004.

[13] S. Wold, N. K. Wold, and B. Skagerberg, "Nonliner pls modeling," Chemometrics and Intelligent laboratory Systems, vol. 7, pp. 53-65, 1989.

[14] S. J. Qin and T. J. McAvoy, "Nonlinear pls modeling using neural networks." Comput. Chem. Eng., vol. 16, no. 4, pp. 379-391, 1992.

[15] D. Lee, M. Lee, S. Woo, Y. Kim, and J. Park, "Nonlinear dynamic partial least squares modeling of a full scale biological wastewater treatment plant." Process Biochemistry, vol. 41, pp. 2050-2057, 1992.

[16] Y. H. Bang, C. K. Yoo, and I.-B. Lee, "Nonliner pls modeling with fuzzy inference system," Chemometrics and Intelligent laboratory Systems, vol. 64, no. 2, pp. 137-155, 2003.

[17] I. Araby, Abdel-Rahman, and G. J. Lim, "A nonliner partial least squares algorithm using quadratic fuzzy system," J. of Chemometrics, vol. 23, pp. 530-537, 2009.

[18] A. Dairi, F. Harrou, Y. Sun, and M. Senouci, "Obstacle detection for intelligent transportation systems using deep stacked autoencoder and $k$-nearest neighbor scheme," IEEE Sensors Journal, vol. 18, no. 12, pp. 5122-5132, 2018.

[19] Y. Wang, Y. Wei, T. Liu, T. Sun, and K. T. Grattan, "Tdlas detection of propane/butane gas mixture by using reference gas absorption cells and partial least square approach," IEEE Sensors Journal, vol. 18, no. 20, pp. 8587-8596, 2018.

[20] J. S. R. Jang, "Anfis-adaptive-network based fuzzy inference system." IEEE Tans. of Systems, man, and Cybernetics, vol. 23, no. 3, pp. 665685, 1993.

[21] — "Fuzzy modeling using generalized neural networks and kalman filtering algorithm," In Proc. Ninth Nat. Conf. Artificial Intell. (AAAI91), pp. 761-767, 1991.

[22] - "Rule extraction using generalized neural networks," Proc. 4th IFSA world congress, vol. 23, no. 3, pp. 191-212, 1991.

[23] A. Wang, N. An, G. Chen, L. Li, and G. Alterovitz, "Accelerating wrapper-based feature selection with k-nearest-neighbor," KnowledgeBased Systems, vol. 83, pp. 81-91, 2015.

[24] X. Wu, V. Kumar, J. R. Quinlan, J. Ghosh, Q. Yang, H. Motoda, G. J. McLachlan, A. Ng, B. Liu, S. Y. Philip et al., "Top 10 algorithms in data mining," Knowledge and information systems, vol. 14, no. 1, pp. $1-37,2008$.

[25] D. C. Montgomery, Introduction to statistical quality control. John Wiley \& Sons, 2007.

[26] E. Martin and A. Morris, "Non-parametric confidence bounds for process performance monitoring charts," Journal of Process Control, vol. 6 , no. 6, pp. 349-358, 1996. 